\title{
The ACL SNNAP Study:
}

\section{ACL Surgery Necessity in Non}

\section{Acute Patients}

\section{Patient Information Sheet}

- We would like to invite you to take part in a research study called ACL SNNAP. The study is looking at the management of anterior cruciate ligament injuries.

- Before you decide whether to take part or not, we would like you to understand why the research is being done and what it would involve for you.

- Please take time to read the following information carefully. If there is anything that is not clear or if you would like more information, please ask us.

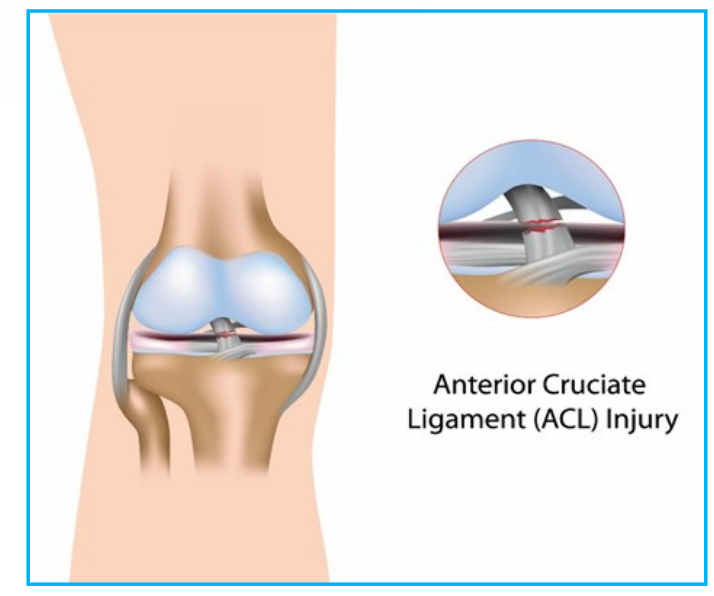

\section{What is the purpose of the ACL SNNAP study?}

The aim of this study is to identify the best approach to treatment for people who have torn a ligament in their knee called the Anterior Cruciate Ligament or ACL.

Currently there are two main NHS clinical management options (commonly used) for this condition;

- Non operative treatment using physiotherapy

- Operative treatment where they reconstruct the ligament

The study will compare these two management options.

Previous studies carried out to help guide treatment decisions for this injury have focused on patients with injuries that have just happened (known as an acute injury). Often patients seen in the NHS will also present with a more longer-standing injury (non-acute injury). It will be these patients with nonacute injuries who will be approached to participate in this study.

The results from this study will help future patients with a non-acute $A C L$ injury decide what treatment to have. 


\section{Why am I being invited to take part?}

You have been invited because you have torn your anterior cruciate ligament, and you are now awaiting treatment.

It has been over 4 months since your injury and you continue to experience symptoms of 'giving way' (instability) of your knee.

We are hoping to recruit over 300 people to the study from NHS hospitals around the UK. We want to understand which package of care is better for patients like you.

\section{Do I have to take part?}

No, taking part in a research study is always optional.

You do not have to decide straightaway. Please feel free to take the information sheet home with you and discuss your participation with friends, family or your own GP.

If you chose not to take part then your clinician will discuss with you the options available for the management of your injury. If you decide not to take part this will not affect the standard of care you will receive.

\section{What will happen if I take part?}

To begin with you will have your knee assessed in the routine clinic visit and be asked to complete a questionnaire relating both to your general health and knee.

If you decide to take part in the study you will undergo one of the following management options:

Physiotherapy - This involves regular physiotherapy sessions under the care of clinicians who specialise in this injury. In these sessions, you will work on improving the strength of your leg muscles, balance and ability to return to activities.

ACL Reconstruction surgery - This operation involves replacing your torn ligament with a graft. The graft is usually taken from a tendon in another part of your knee, for example, your hamstring or patella tendon. Your surgeon will discuss the operation with you and what to expect afterwards in terms of post operative physiotherapy.

All patients participating in this study will attend follow up visits and be monitored at their local hospital as they would normally following physiotherapy or surgery. If any problems are experienced a review appointment will be arranged with the clinical team to discuss future management.

Whichever treatment you receive, you will be asked to complete an email or paper follow up questionnaire at six months; twelve months and eighteen months afterwards. The questionnaires will only ask for information about your injured knee and the associated impact of this on your quality of life. For example questions will ask about whether or not your knee feels stable and if you have restricted your activities because of your knee.

Some patients may also be asked to take part in an interview to discuss their experience of treatment.

$$
2
$$




\section{Which of these management options will I follow?}

We don't know yet. Allocation to these groups is random (like tossing a coin). We are advising this because it is the best way to allow a fair comparison to be made between the different groups. Dividing people into groups in this way is what is called a 'randomised clinical trial' and is the standard and only reliable way to see how good a treatment is. Whatever group you are in, you will still be under the care of your Clinical team and followed up regularly.

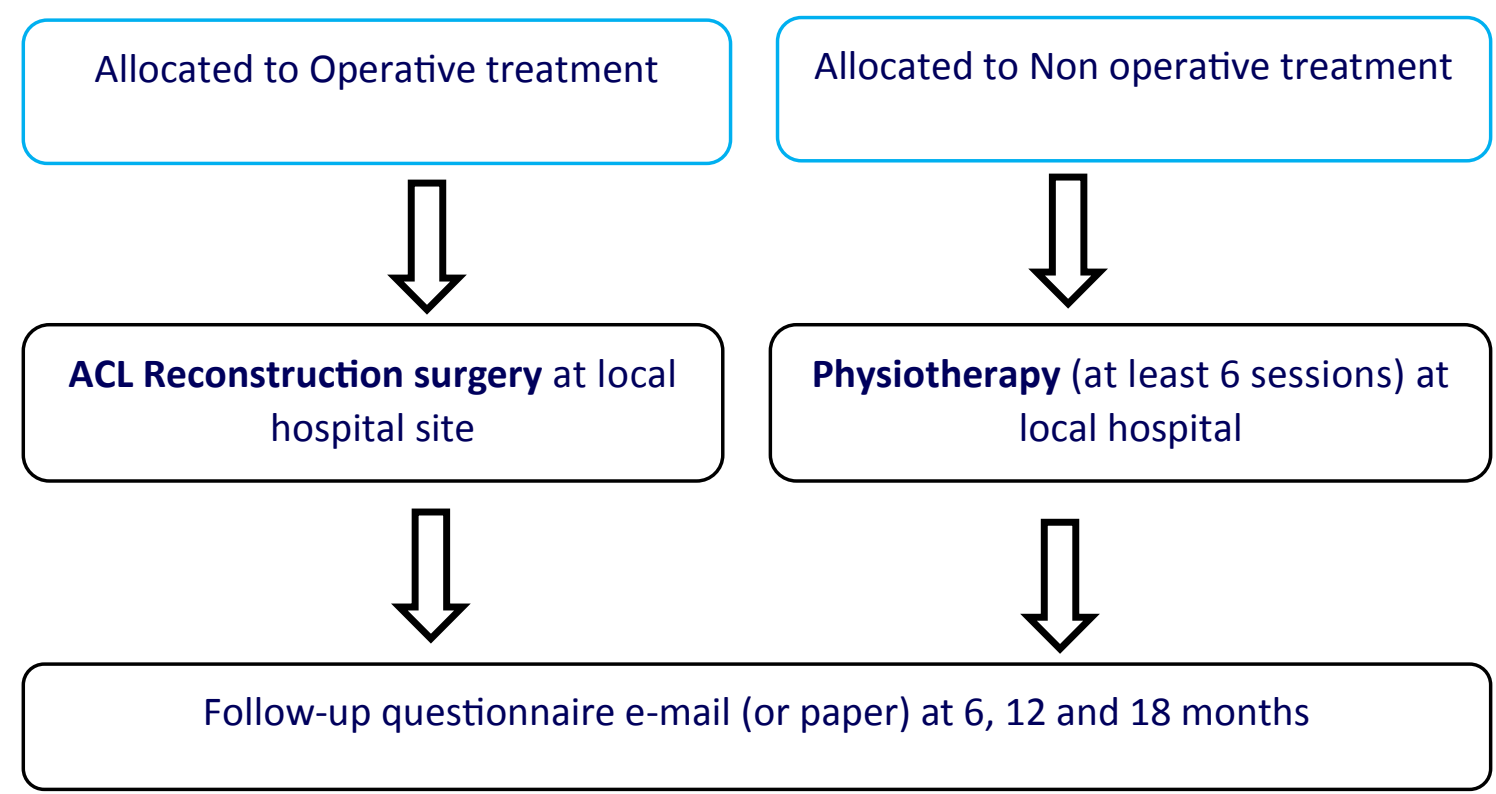

\section{Are there any possible disadvantages or risks from taking part?}

There are no anticipated risks or disadvantages to participating in this study. There are risks associated with any surgery and physiotherapy treatment given in the NHS. These will be discussed with you as part of routine care.

Whichever type of treatment you are allocated to, your care will be the same as if you received this treatment as standard care provided by your local hospital and it will be delivered by a competent and trained clinician.

\section{What are the benefits of taking part?}

We can't guarantee any benefit to patients who take part in this study. Both surgery and nonoperative management are routine care in the NHS and each is a good option for you.

The main benefit from your participation in this study will be the information we get, which should help improve the future treatment of patients who have an ACL injury. 


\section{Will my GP be informed about my participation in this study?}

With your consent we will notify your GP about your participation in this study

\section{Will taking part be kept confidential?}

Yes. All patient information will be stored on password protected computer databases or in locked filing cabinets in line with the University of Oxford and Trust Policy.

You will be allocated a study number and staff not directly involved with your care will know you only by this number.

When the results of the study are reported, individuals who have taken part will not be identified in any way.

Responsible members of the University of Oxford or your NHS Trust may be given access to data for monitoring and/or audit of the study to ensure we are complying with research regulations.

Personal data will be stored for 6-12 months after the study has ended and research data generated by the study will be stored for 5 years in locked filing cabinets accessed only by members of the research team who have card access to the building. After this time this data will be destroyed.

As part of routine care, some surgeons register their patients with the National Ligament Registry (NLR). When you consent to participate in the ACL SNNAP Study, we will also ask you to consent for your information to be shared with the NLR. This will allow us to collaborate with a national database about ACL injuries and treatments. If we can share your information with the NLR then this will prevent you having to answer the same questionnaire from them. Please see the NLR section below.

\section{National Ligament Registry (NLR)}

The NLR was set up with the aim of helping improve the management and outcome of ACL injuries. If your hospital routinely provides data to the NLR then you will consent for this as part of standard care. The NLR routinely follow up patients ion their registry with questionnaires about the pain and function of their knee. More information about the NLR can be found at http://www.uknlr.co.uk.

\section{What will happen if I don't want to carry on with the study?}

Your participation in this study is voluntary and you can choose to leave the study at any time.

If you decide to leave the study, your decision will be respected and this will not affect the care you receive.

\section{Will I be reimbursed for taking part?}

You will not be compensated for your involvement in this study but reasonable travel expenses to the hospital for any appointments that are outside of routine care can be reimbursed. The local research team will provide further details about this. 


\section{What happens at the end of the study?}

At the end of the study all the information we have gathered will be anonymised which means all the patients who have taken part will not be identified. With your consent we would like to make the anonymous data available for use in future research.

The results of the study will be reported to the study funder. This report will be available in the public domain and will also be uploaded onto our website http://www.situ.ox.ac.uk/publications for your information. Some of the research being undertaken will also contribute to the fulfilment of a doctoral thesis.

A summary of the study results with details of where to find further information will be sent to all participants. We also plan to publish the results in a medical journal so others can read about and learn from the results of the study.

\section{What if there is a problem?}

Taking part in the study will not affect your legal rights. If you wish to complain about any aspect of the way in which you have been approached or treated during the course of this study, you should contact your local principal investigator (contact details can be found on the last page of this document).

\section{Or}

Trial Manager at the central office in Oxford by e-mail at acl_snnap@ndorms.ox.ac.uk or by telephone on (01865 227715)

Or

You may contact the University of Oxford Clinical Trials and Research Governance (CTRG) office on 01865 572224, or the head of CTRG, email ctrg@admin.ox.ac.uk.

The Patient Advisory Liaison Service (PALS) is a confidential NHS service that can provide you with support for any complaints or queries you may have regarding the care you receive as an NHS patient at your local hospital. The contact details of your local NHS hospital PALS service can be found below.

[Insert contact details - telephone/address/e-mail of local PALS service]

The University of Oxford, as study Sponsor, has appropriate insurance in place in the unlikely event that you suffer any harm as a direct consequence of your participation in this study. NHS indemnity operates in respect of the clinical treatment which is provided. 


\section{How have patients and the public been involved in this study?}

A Patient and Public Involvement (PPI) group have contributed to the design of the trial and will continue to be involved throughout the study, overseeing the study progress.

\section{Who has reviewed the study?}

To protect your interests, all research in the NHS is looked at by an independent group of people called a Research Ethics Committee. This study has been reviewed and approved by South Central - Oxford C Research Ethics Committee (16/SC/0502). This study has also been reviewed and approved by your local hospital's Research \& Development department.

\section{Who is organising and funding the study?}

The study is organised by a team of surgeons, physiotherapists, nurses, patient representatives, health economists and statisticians working with Professor David Beard and Professor Andrew Price. The team are based at the Nuffield Department of Orthopaedics, Rheumatology \& Musculoskeletal Sciences (NDORMS) at the University of Oxford.

The study is managed by the Surgical Intervention Trials Unit (SITU) and SITU is part of the Oxford Clinical Trials Research Unit (OCTRU). OCTRU is a UKCRC registered Clinical Trials Unit.

At each hospital there will also be a local study team, who will deal directly with your care.

ACL SNNAP is funded by the National Institute of Health Research, Health Technology Assessment Programme (Ref 14/140/63). You can access information about them on their website http:// www.nets.nihr.ac.uk/programmes/hta.

\section{Further information and contact details}

If you have any questions please contact the clinical research team at your local hospital:

Insert Local Hospital Contact Address

You may also contact the Oxford ACL SNNAP team via e-mail at acl snnap@ndorms.ox.ac.uk or telephone number 01865 227715. Information about the study is also available on the SITU website:

\section{Thank you for reading this information and considering taking part in our study.}
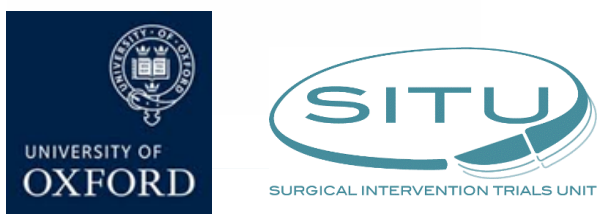